\title{
Novel magnetic stimulation methodology for low-current implantable medical devices
}

\author{
Rodrigo Bernardo ${ }^{a}$, André Rodrigues ${ }^{a}$, \\ Marco P. Soares dos Santos ${ }^{\mathrm{a}, \mathrm{b}, \mathrm{c}, *}$, Pedro Carneiro ${ }^{\mathrm{a}}$, \\ António Lopes $^{\mathrm{d}, \mathrm{e}}$, João Sequeira Amaral ${ }^{\mathrm{d}, \mathrm{e}}$, \\ Vítor Sequeira Amaral ${ }^{\mathrm{d}, \mathrm{e}}$, Raul Morais ${ }^{\mathrm{f}, \mathrm{g}}$ \\ ${ }^{a}$ Department of Mechanical Engineering, University of Aveiro, Aveiro, Portugal. \\ ${ }^{\mathrm{b}}$ Centre for Mechanical Technology 83 Automation (TEMA), University of Aveiro, \\ Aveiro, Portugal. \\ ${ }^{\mathrm{c}}$ Associated Laboratory for Energy, Transports and Aeronautics (LAETA) \\ ${ }^{\mathrm{d}}$ Department of Physics, University of Aveiro, Aveiro, Portugal. \\ e Aveiro Institute of Materials, Aveiro, Portugal. \\ ${ }^{\mathrm{f}}$ University of Trás-os-Montes e Alto Douro, Vila Real, Portugal. \\ ${ }^{\mathrm{g}}$ Institute for Systems and Computer Engineering, Technology and Science \\ (INESC TEC), Porto, Portugal.
}




\begin{abstract}
Recent studies highlight the ability of inductive architectures to deliver therapeutic magnetic stimuli to target tissues and to be embedded into small-scale intracorporeal medical devices. However, to date, current micro-scale biomagnetic devices require very high electric current excitations (usually exceeding 1 A) to ensure the delivery of efficient magnetic flux densities. This is a critical problem as advanced implantable devices demand self-powering, stand-alone and long-term operation. This work provides, for the first time, a novel small-scale magnetic stimulation system that requires up to 50-fold lower electric current excitations than required by relevant biomagnetic technology recently proposed. Computational models were developed to analyse the magnetic stimuli distributions and densities delivered to cellular tissues during in vitro experiments, such that the feasibility of this novel stimulator can be firstly evaluated on cell culture tests. The results demonstrate that this new stimulative technology is able to deliver osteogenic stimuli (0.1-7 mT range) by current excitations in the $0.06-4.3 \mathrm{~mA}$ range. Moreover, it allows coil designs with heights lower than $1 \mathrm{~mm}$ without significant loss of magnetic stimuli capability. Finally, suitable core diameters and stimulator-stimulator distances allow to define heterogeneity or quasi-homogeneity stimuli distributions. These results support the design of high-sophisticated biomagnetic devices for a wide range of therapeutic applications.
\end{abstract}

Key words: Medical device, Biomagnetic device, Implantable device, Magnetic stimulation, Magnetic field

$\bar{*}$ Corresponding author.

Email address: marco.santos@ua.pt (Marco P. Soares dos Santos). 


\section{Introduction}

Research and clinical practice have been highlighting the ability of magnetic stimulation to provide a large variety of diagnostic and therapeutic applications, such as for orthopaedics, neurology, psychiatry and cardiology [1-13]. Biomagnetic stimulation was already approved by well-established national agencies to promote public health by using this non-drug strategy $[4,9,14,15]$. The therapeutic potential of this biophysical approach is also continuously revealed by emerging technologies aiming to develop advanced bioapplications, such as closed-loop bioelectronic implantable devices operating as stand-alone therapeutic agents to treat neurological and psychiatric disorders $[16,17]$. It is also noteworthy to recognize the groundbreaking achievements towards the design of multifunctional bioelectronic devices to provide bone-implant integration and bone regeneration by target-oriented bone stimulation [18-24]. These are examples of advanced implantable devices that require the delivery of personalized stimuli to target tissues. As personalized medicine demand the delivery of highly individualized stimuli $[14,25]$, the superior performance of biomagnetic stimulators will be achieved only if time- and region-dependent magnetic stimuli are delivered, ensuring a wide range of effective stimuli characterized by different waveforms, magnitudes, frequencies, periodicities, stimulation exposures, etc $[14,21,26]$. Different coil designs have been proposed to maximize their performance in the delivery of magnetic stimuli to target tissues, both for extracorporeal $[5,27,28]$ and intracorporeal devices $[1,2,29]$. Electric power requirements is of uppermost importance for intracorporeal biomagnetic devices, as their technological sophistication includes powering ability to supply therapeutic stimulators, as well as physiological sensing and communication systems $[16,17,30]$. However, current small-scale (up to micro-scale) implantable magnetic devices require that high electrical currents (usually exceeding 1 A) must flow in the stimulation coils to ensure the delivery of therapeutic efficient magnetic flux densities [1,2,25,29-31]. This problem has been addressed by using extracorporeal wireless powering [32]; however, this approach presents significant limitations, as it is uncomfortable for patients, it troubles their routine activities and it strongly reduces the periodicity of operation of bioelectronic medical devices [33]. Therefore, as future personalized medicine will demand autonomous stand-alone biomagnetic stimulators with self-powering ability for long-term personalized therapeutic operation $[16,17,21,25,34]$, a new methodology 
is demanded to simultaneously require low electric current excitations and ensure the delivery of suitable magnetic field stimuli. This paper provides, for the first time, a novel small-scale magnetic stimulation technology that requires much lower electric current excitations to deliver the required magnetic fields. Since the feasibility of this innovative device must be predicted prior device implantation and firstly evaluated on cell culture tests, this study is focused on the magnetic stimuli delivered to cellular tissues during in vitro experiments as a function of electric current excitation [25]. Computational models of this novel stimulator are also provided such that magnetic flux densities and distributions are analysed for bone cellular tissues. Nevertheless, breakthroughs here reported will promisingly support biological in vitro and in-vivo experiments using a large variety of tissues. The main goal of this study is to contribute towards the development of implantable high-sophisticated bioelectronic magnetic devices capable of delivering highly controlled and personalized stimuli to a wide range of therapeutic applications.

\section{Methods}

\subsection{Inductive architectures}

The magnetic flux densities that induce positive osteogenic responses are in the 0.1-7 $\mathrm{mT}$ range [35-37]. The ability of planar and ring quasi-planar inductive architectures to provide such stimuli at low power was explored in this study, since they have been used to implement sub-millimeter magnetic stimulators for implantable devices [1,25,29,31]. A planar and two quasi-planar architectures were designed for the delivery of magnetic stimuli to bone cells in culture during the first two stages of bone remodelling (proliferation and differentiation) [25]. The planar architecture (P-architecture), composed by a coil shaped with a planar geometry of spiral pattern and micro-scale wire diameter $(5 \mu \mathrm{m})$, as illustrated by Fig. 1a, was firstly analysed. The ability of a ring quasi-planar architecture ( $\mu \mathrm{M}$-architecture) comprising a cylindrical-shaped core surrounded by a helicoidal-shaped single-layer coil with few turns (20) and micro-scale wire diameter $(5 \mu \mathrm{m})$ to deliver magnetic field stimuli was afterwards explored (Fig. 1b). This architecture and geometrical dimensions are similar to the ones experimentally 
tested by Bonmassar and colleagues $[1,29]$. The manufacture of both $\mathrm{P}$ - and $\mu \mathrm{M}$-architectures for electrophysiological experiments is technically challenging, since they must be small enough to be embedded within implantable devices but ensuring the ability to induce sufficient magnetic flux densities to stimulate tissues $[1,38]$. A new insight is here provided to significantly minimize the electric current supply by using a quasi-planar architecture ( $\mu \mathrm{M}-\mathrm{kT}$-architecture) composed by a cylindrical core surrounded by a helicoidal-shaped multilayered coil with a large number of turns (1000) and micro-scale wire diameter $(5 \mu \mathrm{m})$ (Fig. 1b). This is a more complex and even harder to manufacture architecture compared to already proposed $\mathrm{P}$ - and $\mu \mathrm{M}$-architectures. The proposed methodology is based on the approximative relation of proportionality between the magnetic flux density and the product of the electric current with the number of turns of an electrically excited coil $(B \propto N I)$, obtained from the Ampere's law [39].

\subsection{Simulations details}

All models were implemented considering apparatuses recently validated in silico and in vitro to analyse electromagnetic stimuli throughout proliferation and differentiation stages of osteoblastic MC3T3 cells (a cell line commonly used to analyse bone remodelling stages), which is briefly described as follows $[21,25,26,40]$. This apparatus prohibits cell-electrode contacts and was modelled using seven domains: core, coil, culture medium (a liquid solution), cellular layer (during proliferation stage) or cellular tissue (during differentiation stage), culture dish, substrate and air. Coils and cores were positioned over a polycarbonate substrate and under a polystyrene culture dish. The latter, in turn, contains a cell culture composed of a cellular or a tissue layer and a culture medium (Fig. 1). Remodelling stages were considered approximately homogeneous phases [21]. The cellular/tissue layers were positioned above the culture dish and covered by the liquid solution since osteoblastic MC3T3 cells are highly adherent to polystyrene surfaces $[21,41]$. The thickness of the adherent organic layer was doubled for simulations related to differentiation stage in order to resemble an organized cellular tissue mainly composed by MC3T3 cells and type-I collagen (a protein that corresponds to approximately $90 \%$ of the bone organic matrix) [21]. Polymeric dishes and substrates were used to ensure their very 
high electrical resistivity. The coils were assumed to be made of copper, following previous works $[1,2,25,31]$. The properties of the ferromagnetic cores were taken as those of an Fe-Ni alloy [42]. Table 1 presents all geometric dimensions of each architecture. Table 2 describes electric and magnetic properties of organic and inorganic materials considered to numerically model all architectures. Notice that the geometrical dimensions here explored do not allow only one biomagnetic stimulator to cover the entire area of the bottom surface of the culture dishes [25]. This methodology was chosen in order to highlight the potential of very low scale $\mu \mathrm{M}-\mathrm{kT}$-architectures to deliver osteogenic stimuli at low current excitations.

To compute the magnetic field stimuli generated by these planar and quasi-planar architectures, considering also the present biological architectures, requires a multi-physics approach. The magnetic flux density and distributions were computed by numerical models developed using the AC/DC module of COMSOL Multiphysics (v. 5.3, COMSOL), since this computer simulation tool has been used to analyse electromagnetic stimulation of biological tissues $[1,13,21,40,49]$. The magnetic field stimuli delivered by all inductive architectures to bone cellular tissues during in vitro experiments was simulated using 3 finite element

Table 1

Dimensions of each domain of $P_{-}, \mu M$ - and $\mu M-k T$-architectures of biomagnetic stimulators.

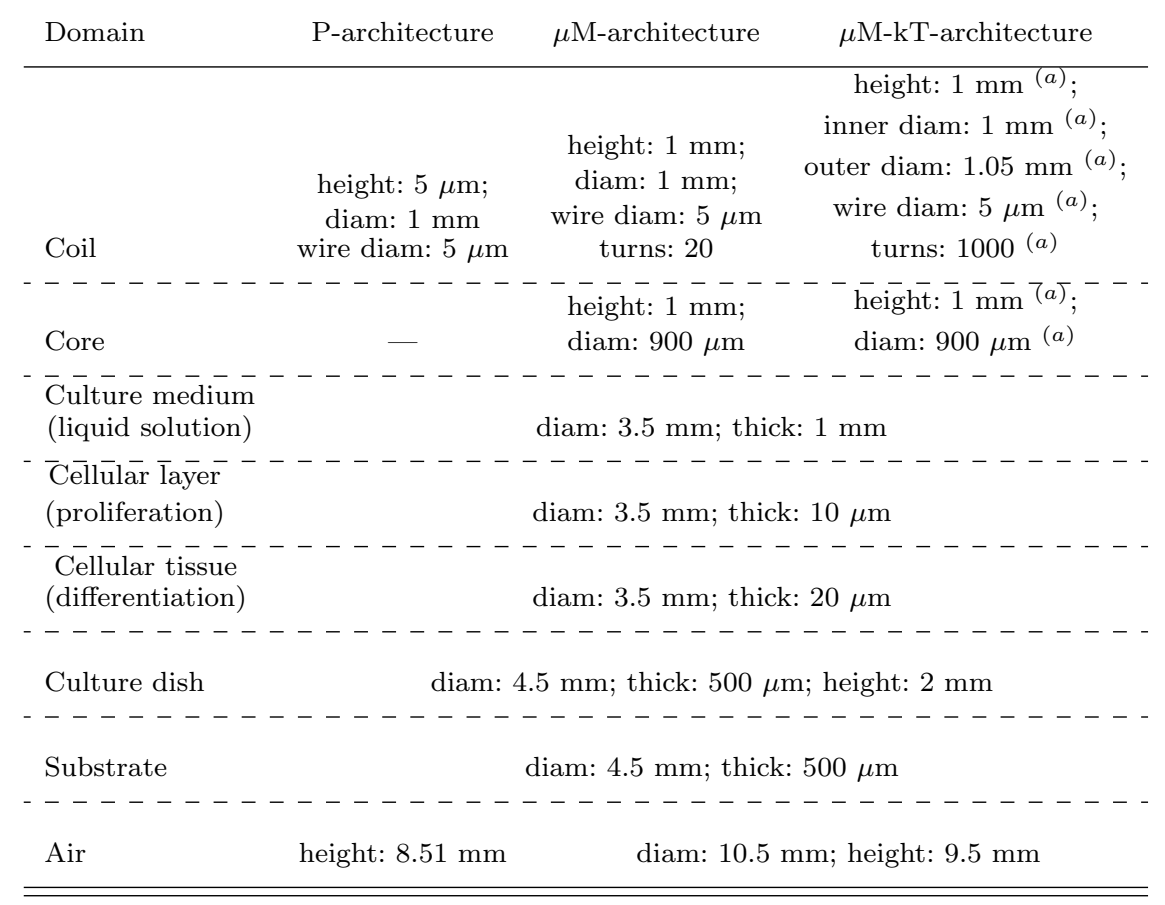

(a) These geometrical dimensions were also varied so that their influence can be quantified. 
Table 2

Electric and magnetic properties of their organic and inorganic materials composing all architectures of biomagnetic stimulators.

\begin{tabular}{|c|c|c|c|c|}
\hline Domain & $\begin{array}{l}\text { Relative electric } \\
\text { permittivity }\end{array}$ & $\begin{array}{l}\text { Electric conductivity } \\
{[\mathrm{S} / \mathrm{m}]}\end{array}$ & $\begin{array}{l}\text { Relative magnetic } \\
\text { permeability }\end{array}$ & Reference \\
\hline $\begin{array}{l}\text { Coil } \\
--------\end{array}$ & $\begin{array}{c}1 \\
-------\end{array}$ & $\begin{array}{c}6 \times 10^{7} \\
--------\end{array}$ & $\begin{array}{c}1 \\
-------\end{array}$ & $\begin{array}{c}{[1,21]} \\
-----\end{array}$ \\
\hline Core & 1 & $1 \times 10^{7}$ & $5 \times 10^{4}$ & {$[42]$} \\
\hline $\begin{array}{l}\text { Culture medium } \\
\text { (liquid solution) }\end{array}$ & 73 & 1.6 & 1 & {$[43,44]$} \\
\hline $\begin{array}{l}\text { Cêllūlär Tayer } \\
\text { (proliferation) }\end{array}$ & $\begin{array}{c}-------- \\
73\end{array}$ & $\begin{array}{c}-------- \\
1.2 \times 10^{-7}\end{array}$ & $\begin{array}{c}-------- \\
1\end{array}$ & {$[43,45]$} \\
\hline $\begin{array}{l}\text { Cellular tissue } \\
\text { (differentiation) } \\
---------\end{array}$ & $\begin{array}{c}73 \\
7-----\end{array}$ & $\begin{array}{c}------- \\
1.2 \times 10^{-7} \\
--------\end{array}$ & $\begin{array}{c}------ \\
1 \\
-------\end{array}$ & $\begin{array}{c}{[46,47]} \\
------\end{array}$ \\
\hline $\begin{array}{l}\text { Culture dish } \\
-------\end{array}$ & 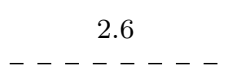 & $\begin{array}{c}6.7 \times 10^{-14} \\
--------\end{array}$ & $\begin{array}{c}1 \\
------.\end{array}$ & $\begin{array}{c}{[21,48]} \\
-----\end{array}$ \\
\hline $\begin{array}{l}\text { Substrate } \\
------.\end{array}$ & $\begin{array}{c}3 \\
------\end{array}$ & $\begin{array}{c}6.7 \times 10^{-14} \\
--------\end{array}$ & $\begin{array}{c}0.866 \\
-------\end{array}$ & $\begin{array}{c}{[48]} \\
-----\end{array}$ \\
\hline Air & 1 & 0 & 1 & {$[1,21]$} \\
\hline
\end{tabular}

computational models: (1) the P-model for the P-architecture (Fig. 1a); (2) the $\mu \mathrm{M}$-model for the $\mu \mathrm{M}$-architecture (Fig. 1b); and (3) the $\mu \mathrm{M}$-kT-model for the $\mu \mathrm{M}$-kT-architecture (Fig. $1 b)$.

All domains were deemed homogeneous and isotropic and were tessellated by fine 3D meshes of tetrahedral linear elements of second order (Delaunay method). Mesh refinement and dimensioning of the 'Air' domain were conducted by convergence analysis ( $2 \%$ error as stop criterion). The homogeneous Neumann condition was imposed to interior boundaries. Null magnetic potential $(\mathbf{A}=0)$ throughout all architecture was established as initial condition. External boundaries were magnetically isolated $(\mathbf{n} \times \mathbf{A}=0)$. Electromagnetic fields were simulated by using 'Magnetic and Electric Fields' as the COMSOL physics interface since it allows to solve the Maxwell's equations in the frequency-domain, as expressed by Eqs. (1) to $(8)$.

$$
\begin{gathered}
\nabla \cdot \mathbf{J}=0 \\
\mathbf{E}=-\nabla \cdot V-\frac{d \mathbf{A}}{d t} \\
\nabla \times \mathbf{H}=\mathbf{J}
\end{gathered}
$$




$$
\begin{gathered}
\mathbf{B}=\nabla \times \mathbf{A} \\
\mathbf{J}=\sigma \mathbf{E}+\frac{d \mathbf{D}}{d t} \\
\mathbf{D}=\varepsilon_{0} \varepsilon_{r} \mathbf{E} \\
\mathbf{B}=\mu_{0} \mu_{r} \mathbf{H} \\
\mathbf{n}_{2} \cdot\left(\mathbf{J}_{1}-\mathbf{J}_{2}\right)=0
\end{gathered}
$$

where: $\mathbf{E}$ - electric field intensity $[\mathrm{V} / \mathrm{m}] ; \mathbf{D}$ - electric displacement $\left[\mathrm{C} / \mathrm{m}^{2}\right] ; \mathbf{H}$ - magnetic field intensity $[\mathrm{A} / \mathrm{m}] ; \mathbf{B}$ - magnetic flux density $[\mathrm{T}] ; \mathbf{J}$ - current density $\left[\mathrm{A} / \mathrm{m}^{2}\right] ; \mathbf{A}$ - magnetic vector potential $[\mathrm{Vs} / \mathrm{m}] ; \sigma$ - electrical conductivity $[\mathrm{S} / \mathrm{m}] ; \varepsilon_{0}$ - permittivity of vacuum $\left(8.85 \times 10^{-12}\right.$ $[\mathrm{F} / \mathrm{m}]) ; \varepsilon_{r}$ - relative permittivity; $\mu_{0}$ - permeability of vacuum $\left(4 \pi \times 10^{-7}[\mathrm{H} / \mathrm{m}]\right) ; \mu_{r}$ - relative permeability; $V$ - electric scalar potential $[\mathrm{V}] ; \mathbf{n}_{2}$ - outward normal from medium 2 at interfaces between two media $\mathbf{J}_{1}$ and $\mathbf{J}_{2} ; \mathbf{J}_{n}$ - current densities of medium $n[\mathrm{~A}]$. The linear FGMRES solver was used to provide fast convergence and computing robustness. All models were computed in a Lenovo P710 workstation with 2 Xeon E5-2690 v4 processors (total 28 physical cores) at $2.6 \mathrm{GHz}$ and $256 \mathrm{~GB}$ RAM.

\subsection{Excitations powering the stimulators}

Coils were powered by an electric current source $I$ in the $0.5-12.5 \mathrm{~mA}$ range, according to the equation: $I=\frac{I_{p p}}{2}+\frac{I_{p p}}{2} \operatorname{sign}(\sin (\omega t))$. The current excitation described throughout this paper refers to the pick-to-pick current $I_{p p}$. Pulsed waveform (50\% duty cycle) and $15 \mathrm{~Hz}$ frequency were selected because many in vitro and in vivo studies highlight the positive osteogenic responses induced by these stimuli parameters using magnetic stimulation throughout different daily exposure time and days of exposure [7-9,37,50,51].

\subsection{Data analysis}

The origin plane $(x, y, 0)$ match the upper boundary of stimulators' coil. The magnetic field was analysed along a $x y$-plane in a vertical $z$-coordinate corresponding to the cellular layer/tissue 
midpoint: along $(x, y, 0.505)[\mathrm{mm}]$ for the stimuli delivered throughout the proliferation stage, and $(x, y, 0.51)[\mathrm{mm}]$ for the differentiation stage. The magnetic fields were also observed along the line $y=0[\mathrm{~mm}]$ (i.e, along $(x, 0,0.505)[\mathrm{mm}]$ for proliferation and $(x, 0,0.51)[\mathrm{mm}]$ for differentiation). Section 'Results' only presents the magnetic flux density at $\pi / 2 \mathrm{rad}$, as these stimuli exhibit pulsed electromagnetic fields (PEMF) dynamics (since cores were defined as soft magnetic materials).

\section{Results}

\subsection{Influence of cell confluence}

The magnetic flux densities and distributions along the cellular layer $\left(z \in\left[\begin{array}{lll}0.5 & 0.51\end{array}\right][\mathrm{mm}]\right.$; low cell confluence condition; proliferation stage) and along the cellular tissue $(z \in[0.50 .52][\mathrm{mm}]$; full cell confluence condition; matrix maturation stage) are quite similar. Cross-correlations of nearly $100 \%$ and amplitude differences lower than $1 \%$ were perceived. Thereupon, only the stimuli delivered to bone cells throughout proliferation will be analysed in the following sections, i.e., along $(x, y, 0.505)[\mathrm{mm}]$.

\subsection{Influence of the architecture and current excitation}

Similar heterogeneous distributions of magnetic stimuli (cross-correlations higher than 99\%) are delivered by biomagnetic stimulators to the bone cellular tissue when their coils are powered by the same electric current source, imposing maximum magnetic flux densities in regions above the cores (Fig. 2). Nevertheless, quite differing magnetic flux densities are observed. The $\mu \mathrm{M}-\mathrm{kT}$-architecture is able to provide approximately $4 \mathrm{mT}$ for $2.5 \mathrm{~mA}$ current excitation (Fig. 2c,d). This is a magnetic stimulus approximately 50-fold and 34-fold higher than the stimuli delivered by $\mu \mathrm{M}$ - and P-architectures, respectively (Fig. 2a,b,d). The architecture-dependent stimulation is also noticeable by analysing the required current excitation to provide osteogenic stimuli: magnetic flux densities in the 0.1-7 $\mathrm{mT}$ range are obtained by exciting the $\mu \mathrm{M}-\mathrm{kT}$-architecture using an electric current in the $0.06-4.3 \mathrm{~mA}$ 
range (Fig. 2e,f); in contrast, the same magnetic stimuli range will require powering the $\mu \mathrm{M}$ - and P-architectures, respectively, with approximately 3.1-212.6 mA and 2.1-146.4 mA. Linear interrelationships occur, as expected, between current excitations supplying coils and magnetic densities delivered along the overall cell tissue (slope $B[\mathrm{mT}] / i[\mathrm{~mA}]$ is 1.62 for the $\mu \mathrm{M}$-kT-architecture, $32.9 \times 10^{-3}$ for the $\mu \mathrm{M}$-architecture and $47.8 \times 10^{-3}$ for the P-architecture), as illustrates Fig. 2f. All these results emphasize that further analyses must be conducted to identify the effects of varying the physical properties of the $\mu \mathrm{M}$-kT-architecture, namely the influence of the coil and core, as well as stimulators positioning. So, next sections will be focused on the influence of these design parameters for magnetic stimulation performed by the $\mu \mathrm{M}-\mathrm{kT}$-architecture.

\subsection{Influence of the coil and core}

The influence of the number of turns is of utmost importance for the design of biomagnetic devices. Fig. 3a highlights the magnetic flux densities delivered by $\mu \mathrm{M}-\mathrm{kT}$-architecture as a function of the number of turns. Minimum osteogenic magnetic stimulus $(0.1 \mathrm{mT})$ will be obtained by using 25 turns (2.5 mA current excitation). Nevertheless, most in vitro and in vivo studies propose magnetic flux densities higher than $1 \mathrm{mT}$ for a successful stimulation therapy, which requires 250 turns (Fig. 3b). A coil comprising 1750 turns must be designed for the maximum flux density (7 $\mathrm{mT})$ to be delivered to cellular tissues (Fig. 3b). Although the use of different number of turns results in different stimuli magnitudes (if the current excitation is not varied), the stimuli distribution waveforms along the cellular tissue are quite similar (cross-correlation of nearly 100\%).

No significant impacts are predicted on distributions and densities of magnetic flux when

different designs are established by varying the coil and core heights (Fig. 4a). Similarity on stimuli distribution waveforms are observed when the coil height is varied in the 100-1000 $\mu \mathrm{m}$ range (cross-correlations higher than 99\%). One must, however, recognize that even a stimulator designed with a $100 \mu \mathrm{m}$ coil height is able to deliver osteogenic magnetic stimuli (approximately $3.7 \mathrm{mT}$ ) when it is driven by $2.5 \mathrm{~mA}$ current source. 
Cross-correlations in magnetic stimuli distributions of approximately $99.6 \%$ were observed for core diameters lower than $900 \mu \mathrm{m}$, but the lower the core diameter the lower maximum magnetic flux densities provided by the $\mu \mathrm{M}$-kT-architecture based stimulator (Fig. $4 \mathrm{~b}$ ). Nevertheless, no significant changes on the stimuli magnitudes were observed for varying core diameters, since they are mainly influenced by the number of coil turns and the electric current excitation (as predicted by the Ampere's law). Hence, the design of biomagnetic stimulators with core diameters lower than $500 \mu \mathrm{m}$ are affective to deliver osteogenic stimuli using $2.5 \mathrm{~mA}$ current excitation. A quite different behaviour is perceived for core diameters higher than 1 $\mathrm{mm}$ : distribution similarity decrease to $91.3 \%$ and the maximum magnetic flux decrease $7.3 \%$ when $900 \mu \mathrm{m}$ and $2 \mathrm{~mm}$ core diameters are compared. Besides, the maximum magnetic flux is horizontally shifted on the cellular tissue above the core to above the coils.

\subsection{Influence of the stimulators positioning}

Due to the very small geometric dimensions of the $\mu \mathrm{M}-\mathrm{kT}$-architecture, in vitro experimental tests will require several biomagnetic stimulators delivering magnetic stimuli to cellular layers/tissues. Their relative positioning to each other allow the delivery of magnetic stimuli to tissue areas in both quasi-homogeneous and heterogeneous manner. The influence of the equidistances between the geometric center of two cores is highlighted in Fig. 5. Quasi-homogeneous magnetic flux densities are provided for very short stimulator-stimulator distances (as it approaches $1.06 \mathrm{~mm}$ ), whereas heterogeneity increases for increasing distances. Notice the decreasing magnetic flux density around the midpoint between geometric centers for increasing stimulator-stimulator distance. Null magnetic stimulus is delivered in this midpoint for distances higher than $3 \mathrm{~mm}$. Finally, it is noteworthy to observe that the experimental apparatus to conduct both in vitro and in vivo tests will require a network of independent biomagnetic stimulators. For instance, if only 10 stimulators are demanded to deliver a $1 \mathrm{mT}$ PEMF stimulative therapy along a target tissue, then the self-powering system must provide approximately $6.2 \mathrm{~mA}$ current excitation if the $\mu \mathrm{M}$-kT-stimulator is used; however, $209 \mathrm{~mA}$ and $304 \mathrm{~mA}$ will be required for the $\mathrm{P}$-stimulator and $\mu \mathrm{M}$-stimulator, respectively. 


\section{Discussion and conclusion}

Recent research findings have highlighted the therapeutic potential of contactless biomagnetic stimulators for delivery of personalized non-drug therapies to target tissues $[1,25,29]$. Low power and electric current requirements are mandatory for innovative implantable medical devices, mainly if they provide multifunctional operation (therapeutic stimulation, sensing and communication ability). Several analyses were recently conducted to identify the influence of the relevant planar and quasi-planar architectures on the deliver of magnetic stimuli, as well as the influence of some of their physical properties $[1,40]$. However, these studies were focused on $\mu \mathrm{M}$-architectures, and usually electric currents exceeding $1 \mathrm{~A}$ are required to drive micro-inductors. This work is focused on the ability of a novel quasi-planar architecture for small-scale implantable devices, the $\mu \mathrm{M}-\mathrm{kT}$-architecture, to induce sufficient magnetic flux densities to tissue target by powering coils by low current excitations. By developing numerical models using COMSOL Multiphysics to compute the magnetic stimuli delivered to osteoblastic MC3T3 cells throughout in vitro experimental tests, significant differences in electric current excitations required to induce positive osteogenic responses (in the $0.1-7 \mathrm{mT}$ range) were observed. The architecture here proposed is able to provide such magnetic stimuli if currents in the 0.06-4.3 mA range flow through the coil. The greater complexity of the $\mu \mathrm{M}$-kT-architecture must be noticed, but it requires 50-fold and 34-fold lower current excitations than $\mu \mathrm{M}-$ and $\mathrm{P}$-architectures, respectively. Moreover, this quasi-planar $\mu \mathrm{M}-\mathrm{kT}$-architecture can be redesigned to include sophisticated coil heights lower than $500 \mu \mathrm{m}$ without significant magnetic stimuli decreases, which is another advantage over P-architectures. Besides, heterogeneous or quasi-homogeneous magnetic stimuli distributions can be established by defining appropriated core diameters and the stimulator-stimulator distance.

These analyses were firstly considered for cell culture tests in which the target is a cellular tissue. In vitro stimulation of cellular tissues on cell culture dishes of $35 \mathrm{~mm}$ in diameter will require a network of $\approx 300 \mu \mathrm{M}-\mathrm{kT}$ stimulators ( $3 \mathrm{~mm}$ equidistance between adjacent cores; Fig. 5; Table 1) and, consequently, $18.6 \mathrm{~mA}$ of electric current excitation will be demanded if each stimulator is independently driven with $0.06 \mathrm{~mA}$ (Fig. 2f). Such solution is suitable for biomagnetic devices that must be designed to deliver different stimuli to very close target 
regions of the same tissue. However, lower electric current excitations will be demanded if: (i) arrays of $\mu \mathrm{M}-\mathrm{kT}$ stimulators are electrically connected in series circuit; (ii) a network of larger $\mu \mathrm{M}-\mathrm{kT}$ stimulators is used. This latter described apparatus will only require $1.8 \mathrm{~mA}$ to deliver osteogenic stimuli along the cellular tissue if $9 \mu \mathrm{M}-\mathrm{kT}$ stimulators, with $6 \mathrm{~mm}$ of core diameter, are driven with $0.2 \mathrm{~mA}$ of current excitation, as illustrated by Fig. 6. Notice that the network of stimulators could be designed using coils with different diameters, such that they can cover almost the entire area of the bottom surface of the culture dishes.

Further research must be conducted for much more complex bone structures, such as trabecular and cortical structures comprising liquid, organic and mineral phases. Besides, the impact of the $\mu \mathrm{M}-\mathrm{kT}$-architecture must also be evaluated for a wide range of biological structures, including for neurological and psychiatric applications. Temperature and loss effects must also be considered in future research works in this scope. The ultimate goal is to support the design of innovative active biomagnetic medical devices based on biophysical stimulation and their future clinical translation to tissues or organs therapies of patients.

\section{Acknowledgement}

This work was funded by the Portuguese Foundation for Science and Technology (FCT), through the grant references SFRH/BPD/117475/2016, SFRH/BD/129340/2017 and IF/01089/2015, and by the European Structural and Investment Funds, through the project reference POCI-01-0145-FEDER-031132 and POCI-01-0145-FEDER-007679. It was also support by the TEMA - Centre for Mechanical Technology \& Automation (UID/EMS/00481/2013-FCT and CENTRO-01-0145-FEDER-022083) and CICECO - Aveiro Institute of Materials (UID /CTM /50011/ 2013).

\section{Author contributions}

Rodrigo Bernardo, André Rodrigues and Marco P. Soares dos Santos contributed equally to this work. 


\section{Additional information}

Conflicts of interests: None

Competing funding: None

Ethical approval: Not required

\section{References}

[1] G. Bonmassar, S. Woo, D. K. Freeman, M. Polasek, S. I. Fried, J. T. Gale, Microscopic magnetic stimulation of neural tissue, Nature Communications 3 (2012) 921.

[2] S. W. Lee, F. Fallegger, B. D. F. Casse, S. I. Fried, Implantable microcoils for intracortical magnetic stimulation, Science Advances 2 (12) (2016) e1600889.

[3] S. Luan, I. Williams, K. Nikolic, T. G. Constandinou, Neuromodulation: present and emerging methods, Frontiers in Neuroengineering 7 (2014) 27.

[4] P. M. Lewis, R. H. Thomson, J. V. Rosenfeld, P. B. Fitzgerald, Brain neuromodulation techniques: A review, The Neuroscientist 22 (4) (2016) 406-421.

[5] L. J. Crowther, P. Marketos, P. I. Williams, Y. Melikhov, D. C. Jiles, J. H. Starzewski, Transcranial magnetic stimulation: Improved coil design for deep brain investigation, Journal of Applied Physics 109 (2011) 07B314.

[6] L. J. Crowther, I. C. Nlebedim, D. C. Jiles, Developments in deep brain stimulation using time dependent magnetic fields, Journal of Applied Physics 111 (2012) 07B325 .

[7] Z. He, N. Selvamurugan, J. Warshaw, N. C. Partridge, Pulsed electromagnetic elds inhibit human osteoclast formation and gene expression via osteoblasts, Bone 106 (2018) 194-203.

[8] T. Lei, Z. Liang, F. Li, C. Tang, K. Xie, P. Wang, X. Dong, S. Shan, M. Jiang, Q. Xu, E. Luo, G. Shen, Pulsed electromagnetic elds (PEMF) attenuate changes in vertebral bone mass, architecture and strength in ovariectomized mice, Bone 108 (2018) 10-19. 
[9] E. I. Waldorff, N. Zhang, J. T. Ryaby, Pulsed electromagnetic eld applications: A corporate perspective, Journal of Orthopaedic Translation 9 (2017) 60-68.

[10] L. Petecchia, F. Sbrana, R. Utzeri, M. Vercellino, C. Usai, L. Visai, M. Vassalli, P. Gavazzo, Electro-magnetic field promotes osteogenic differentiation of BM-hMSCs through a selective action on $\mathrm{ca}^{2+}$-related mechanisms, 20155 (Scientific Reports) 13856.

[11] J. Wang, Y. An, F. Li, D. Li, D. Jing, T. Guo, E. Luo, C. Ma, The effects of pulsed electromagnetic eld on the functions of osteoblasts on implant surfaces with different topographies, Acta Biomaterialia 10 (2) (2014) 975-985.

[12] Z. B. Kagan, A. K. RamRakhyani, G. Lazzi, R. A. Normann, D. J. Warren, In vivo magnetic stimulation of rat sciatic nerve with centimeter- and millimeter-scale solenoid coils, IEEE Transactions on Neural Systems and Rehabilitation Engineering 24 (11) (2016) $1138-1147$.

[13] H.-J. Park, J. H. Seol, J. Ku, S. Kim, Computational study on the thermal effects of implantable magnetic stimulation based on planar coils, IEEE Transactions on Biomedical Engineering 63 (1) (2016) 158-167.

[14] J.-P. Lefaucheur, N. André-Obadia, A. Antal, S. S. Ayache, C. Baeken, D. H. Benninger, R. M. Cantello, M. Cincotta, M. de Carvalho, D. D. Ridder, H. Devanne, V. D. Lazzaro, S. R. Filipovic, F. C. Hummel, S. K. J. ad Vasilios K. Kimiskidis, G. Koch, B. Langguth, T. Nyffeler, A. Oliviero, F. Padberg, E. Poulet, S. Rossi, P. M. Rossini, J. C. Rothwell, C. Schönfeldt-Lecuona, H. R. Siebner, C. W. Slotema, C. J. Stagg, J. Valls-Sole, U. Ziemann, W. Paulus, L. Garcia-Larrea, Evidence-based guidelines on the therapeutic use of repetitive transcranial magnetic stimulation (rTMS), Clinical Neurophysiology 125 (11) (2014) 2150-2206.

[15] M. Griffin, A. Bayat, Electrical stimulation in bone healing: Critical analysis by evaluating levels of evidence, ePlasty 11 (2011) e34.

[16] G. Panuccio, M. Semprini, M. Chiappalone, Intelligent biohybrid systems for functional brain repair, New Horizons in Translational Medicine 3 (3-4) (2016) 162-174. 
[17] G. Panuccio, M. Semprini, L. Natale, S. Buccelli, I. Colombi, M. Chiappalone, Progress in neuroengineering for brain repair: New challenges and open issues, Brain and Neuroscience Advances 2 (2018) 1-11.

[18] M. P. Soares dos Santos, J. A. F. Ferreira, A. Ramos, J. A. O. Simões, Active orthopaedic implants: Towards optimality, Journal of the Franklin Institute 352 (3) (2015) 813-834.

[19] C. Schmidt, U. Zimmermann, U. van Rienen, Modeling of an optimized electrostimulative hip revision system under consideration of uncertainty in the conductivity of bone tissue, IEEE Journal of Biomedical and Health Informatics 19 (4) (2015) 1321-1330.

[20] U. Zimmermann, U. van Rienen, An automatic pareto classier for the multiobjective optimization of an electrostimulative acetabular revision system, IEEE Transactions on Magnetics 50 (2) (2014) 7018304.

[21] M. P. Soares dos Santos, A. Marote, T. Santos, J. Torrão, A. Ramos, J. A. O. Simões, O. A. B. da Cruz e Silva, E. P. Furlani, S. I. Vieira, J. A. F. Ferreira, New cosurface capacitive stimulators for the development of active osseointegrative implantable devices, Scientific Reports 6 (2016) 30231.

[22] J. N. D. Torrão, M. P. S. dos Santos, J. A. F. Ferreira, Instrumented knee joint implants: innovations and promising concepts, Expert Reviews of Medical Devices 12 (5) (2015) $571-584$

[23] M. P. Soares dos Santos, J. A. F. Ferreira, A. Ramos, J. A. O. Simões, R. Morais, N. M. Silva, P. M. Santos, M. C. Reis, T. Oliveira, Instrumented hip joint replacements, femoral replacements and femoral fracture stabilizers, Expert Reviews of Medical Devices 11 (6) (2014) 617-635.

[24] N. M. Silva, P. M. Santos, J. A. F. Ferreira, M. P. Soares dos Santos, A. Ramos, J. A. O. Simões, M. J. C. S. R. ad Raul Morais, Power management architecture for smart hip prostheses comprising multiple energy harvesting systems, Sensors and Actuators A: Physical 202 (2013) 183-192.

[25] S. Grehl, D. Martina, C. Goyenvalle, Z.-D. Deng, J. Rodger, R. M. Sherrard, In vitro magnetic stimulation: A simple stimulation device to deliver dened low intensity electromagnetic fields, Frontiers in Neural Circuits 10 (2016) 85. 
[26] M. P. S. dos Santos, J. Coutinho, A. Marote, B. Sousa, A. Ramos, J. A. F. Ferreira, R. Bernardo, A. Rodrigues, A. T. Marques, O. A. B. da Cruz e Silva, J. A. O. S. Edward P. Furlani, S. I. Vieira, Capacitive technologies for highly controlled and personalized electrical stimulation by implantable biomedical systems, Scientific Reports, 9 (2019) 5001.

[27] V. Guadagnin, M. Parazzini, S. Fiocchi, I. Liorni, P. Ravazzani, Deep transcranial magnetic stimulation: Modeling of different coil congurations, IEEE Transactions on Biomedical Engineering 63 (7) (2016) 1543-1550.

[28] Z.-D. Deng, S. H. Lisanby, A. V. Peterchev, Coil design considerations for deep transcranial magnetic stimulation, Clinical Neurophysiology 125 (6) (2014) 1202-1212.

[29] H.-J. Park, G. Bonmassar, J. A. Kaltenbach, A. G. Machado, N. F. Manzoor, J. T. Gale, Activation of the central nervous system induced by micro-magnetic stimulation, Nature Communications 4 (2013) 2463.

[30] P. M. Rossini, L. Rossini, F. Ferreri, Brain-behavior relations: Transcranial magnetic stimulation: A review, IEEE Engineering in Medicine and Biology Magazine 29 (1) (2010) $84-95$.

[31] T. Pashut, D. Magidov, H. Ben-Porat, S. Wolfus, A. Friedman, E. Perel, M. Lavidor, I. Bar-Gad, Y. Yeshurun, A. Korngreen, Patch-clamp recordings of rat neurons from acute brain slices of the somatosensory cortex during magnetic stimulation, Frontiers in Cell. Neurosci 8 (2014) 145.

[32] A. R. Burton, P. Sun, J. P. Lynch, Bio-compatible wireless inductive thin-film strain sensor for monitoring the growth and strain response of bone in osseointegrated prostheses, Structural Health Monitoring (2019) in press.

[33] M. P. Soares dos Santos, J. A. F. Ferreira, A. Ramos, J. A. O. Simões, R. Morais, N. M. Silva, P. M. Santos, M. J. C. S. Reis, T. Oliveira, Instrumented hip implants: Electric supply systems, Journal of Biomechanics 46 (15) (2013) 2561-2571.

[34] M. P. Soares dos Santos, J. A. F. Ferreira, J. A. O. Simões, R. Pascoal, J. Torrão, X. Xue, E. P. Furlani, Magnetic levitation-based electromagnetic energy harvesting: a semi-analytical non-linear model for energy transduction, Scientific Reports 6 (18579) (2016) . 
[35] K. S. Kang, J. M. Hong, J. A. Kang, J.-W. Rhie, Y. H. Jeong, D.-W. Cho, Regulation of osteogenic differentiation of human adipose-derived stem cells by controlling electromagnetic field conditions, Experimental \& Molecular Medicine 45 (2013) e6.

[36] K. Heermeier, M. Spanner, J. Trager, R. Gradinger, P. G. Strauss, W. Kraus, J. Schmidt, Effects of extremely low frequency electromagnetic field (EMF) on collagen type i mRNA expression and extracellular matrix synthesis of human osteoblastic cells, Bioelectromagnetics 19 (4) (1998) 222-231.

[37] P. Diniz, K. Shomura, K. Soejima, G. Ito, Effects of pulsed electromagnetic field (PEMF) stimulation on bone tissue like formation are dependent on the maturation stages of the osteoblasts, Bioelectromagnetics 23 (5) (2002) 398-405.

[38] N. Muggleton, V. Walsh, Smaller magnets for smarter minds?, trends in Cognitive Sciences 16 (9) (2012) 452-453.

[39] A. E. Umenei, Y. Melikhov, D. C. Jiles, Analytic solution for variations of magnetic fields in closed circuits:examination of deviations from the standard Ampere's law equation, IEEE Transactions of Magnetics 47 (4) (2011) 734-737.

[40] G. Bonmassar, J. Gale, W. Vanduffel, Optimizing microscopic magnetic fields for neuronal stimulation, International Journal of Bioelectromagnetism 16 (1) (2014) 1-31.

[41] S. Lavenus, P. Pilet, J. Guicheux, P. Weiss, G. Louarn, P. Layrolle, Behaviour of mesenchymal stem cells, fibroblasts and osteoblasts on smooth surfaces, Acta Biomaterialia 7 (4) (2011) 1525-1534.

[42] J. A. Bas, J. A. Calero, M. J. Dougan, Sintered soft magnetic materials. properties and applications, Journal of Magnetism and Magnetic Materials 254-255 (2003) 391-398.

[43] H. Ozawa, E. Abe, Y. Shibasaki, T. Fukuhara, T. Suda, Electric fields stimulate DNA synthesis of mouse osteoblast-like cells (MC3T3-El) by a mechanism involving calcium ions, Journal of Cellular Physiology 138 (3) (1989) 477-483.

[44] G. Pucihar, T. Kotnik, M. Kandušer, D. Miklavčič, The influence of medium conductivity on electropermeabilization and survival of cells in vitro, Bioelectrochemistry 54 (2) (2001) 107-115. 
[45] H.-P. Wiesmann, M. Hartig, U. Stratmann, U. Meyer, U. Joos, Electrical stimulation influences mineral formation of osteoblast-like cells in vitro, Biochimica et Biophysica Acta (BBA) - Molecular Cell Research 1538 (1) (2001) 28-37.

[46] V. P. Tomaselli, M. H. Shamos, Electrical properties of hydrated collagen. I. dielectric properties, Biopolymers 12 (2) (1973) 353-366.

[47] V. P. Tomaselli, M. H. Shamos, Electrical properties of hydrated collagen. II. semiconductor properties, Biopolymers 13 (12) (1974) 2423-2434.

[48] J. Brandup, E. H. Immergut, E. A. Grulke (Eds.), Polymer Handbook, 4th Edition, John Wiley and Sons, New York, 1999.

[49] H. Cho, Y.-K. Seo, H.-H. Yoon, S.-C. Kim, S.-M. Kim, K.-Y. Song, J.-K. Park, Neural stimulation on human bone marrow-derived mesenchymal stem cells by extremely low frequency electromagnetic fields, Biotechnology Progress 28 (5) (2012) 1329-1335.

[50] C. H. Lohmann, Z. Schwartz, Y. Liu, H. Guerkov, D. D. Dean, B. Simon, B. D. Boyan, Pulsed electromagnetic field stimulation of MG63 osteoblast-like cells affects differentiation and local factor production, Journal of Orthopaedic Research 18 (4) (2000) $637-646$.

[51] C. H. Lohmann, Z. Schwartz, Y. Liu, Z. Li, B. J. Simon, V. L. Sylvia, D. D. Dean, L. F. Bonewald, H. J. Donahue, B. D. Boyan, Pulsed electromagnetic fields affect phenotype and connexin 43 protein expression in MLO-Y4 osteocyte-like cells and ROS 17/2.8 osteoblast-like cells., Journal of Orthopaedic Research 21 (2) (2003) 326-334. 
Fig. 1. Computational models of inductive architectures: (a) P-model for the P-architecture; (b) $\mu \mathrm{M}$-model for the $\mu \mathrm{M}$-architecture, and $\mu \mathrm{M}$-kT-model for the $\mu \mathrm{M}-\mathrm{kT}$-architecture. Domains: 1 - Culture medium (a liquid solution); 2 - Cellular layer (proliferation stage) or cellular tissue (differentiation stage); 3 - Culture dish; 4 - Coil (quasi-planar coils: single-layer coil for $\mu \mathrm{M}$-architectures; multilayered coil for $\mu \mathrm{M}$-kT-architectures); 5 - Polymeric substrate; 6 - Air; 7 - Core.

Fig. 2. Magnetic flux density delivered by biomagnetic stimulators to bone cellular tissue for $5 \mathrm{~mA}$ current excitation along $(x, y, 0.505) \mathrm{mm}$ : (a) P-architecture; (b) $\mu \mathrm{M}$-architecture; (c) $\mu \mathrm{M}-\mathrm{kT}$-architecture. (d) Magnetic flux density along $(x, 0,0.505) \mathrm{mm}$ delivered by all architectures. Magnetic flux density along $(x, y, 0.505)$ for differing current excitations: (e) 1 , 5 and $10 \mathrm{~mA}$ current excitation applied to drive the $\mu \mathrm{M}-\mathrm{kT}$-architecture; (f) Magnetic flux densities delivered by all architectures as a function of current excitation. The dashed line represent the geometric center of the stimulator $(x=3 \mathrm{~mm})$.

Fig. 3. Magnetic flux density delivered by $\mu \mathrm{M}-\mathrm{kT}$-architecture for $5 \mathrm{~mA}$ current excitation along $(x, y, 0.505) \mathrm{mm}$ : (a) influence of a coil design comprising 20, 500, 1000 and 2000 turns (the coil outer diameter was also redesigned to allow this analysis); (b) Magnetic flux densities as a function of the number of turns. The dashed line represent the geometric center of the stimulator $(x=3 \mathrm{~mm})$.

Fig. 4. Magnetic flux density delivered by $\mu \mathrm{M}-\mathrm{kT}$-architecture for $5 \mathrm{~mA}$ current excitation along $(x, y, 0.505) \mathrm{mm}$ : (a) influence of $100 \mu \mathrm{m}, 500 \mu \mathrm{m}$ and $1 \mathrm{~mm}$ coil heights (the coil outer diameter was also redesigned to allow this analysis); (b) influence of $500 \mu \mathrm{m}, 900 \mu \mathrm{m}$ and 2 $\mathrm{mm}$ core diameters. The dashed line represent the geometric center of the stimulator $(x=3$ $\mathrm{mm})$.

Fig. 5. Influence of the stimulators positioning on the distribution of magnetic stimuli along $(x, y, 0.505) \mathrm{mm}$ provided by two biomagnetic stimulators based on $\mu \mathrm{M}-\mathrm{kT}$-architecture.

Fig. 6. In vitro stimulation on cell culture dishes of $35 \mathrm{~mm}$ in diameter using $9 \mu \mathrm{M}-\mathrm{kT}$ stimulators with $6 \mathrm{~mm}$ of core diameter, $1 \mathrm{~mm}$ of height and 1000 coil turns, when driven with $0.2 \mathrm{~mA}$ of current excitation. 